\title{
Research on the Optimization of a Drilling Rock Breaking Method Based on Fuzzy Cluster Analysis
}

\author{
Kun Du and Zhen Wei \\ Shengli Petroleum Engineering Co., Ltd. of Sinopec, Dongying, 257100, China \\ *Corresponding Author: Zhen Wei. Email: weizhen107chn@163.com \\ Received: 29 September 2021 Accepted: 26 October 2021
}

\begin{abstract}
Improving drilling efficiency is the best way to reduce drilling costs and the choice of the drilling mode is instrumental in doing so. At present, however, a standard approach for the optimization of these processes does not exists yet. Through a comparative statistical analysis of the rock-breaking mechanisms and the characteristics of different drilling methods, this research proposes a set of cues to achieve this objective. Available statistical data are classified by means of a fuzzy cluster analysis according to the anti-drilling characteristic parameters of formation. The results show that different drilling methods rely on their own rock breaking mechanisms and have distinct characteristics. The rotary table drilling method is the most commonly used drilling mode, however, it displays some limitations with regard to deep wells, ultra-deep wells and difficult formations. The combined drilling method has the advantages of both the rotary table drilling and the down-hole power drilling modes. Polycrystalline diamond compact (PDC) drill bits can lead to good results for medium hardness and weakly abrasive formations. Underbalanced drilling for formations with high hardness and strong abrasiveness displays some limitations.
\end{abstract}

\section{KEYWORDS}

Drilling methods; anti-drilling characteristic; fussy clustering; optimization method

\section{Introduction}

With a long period of development, the methods of oil and gas drilling have been basically become mature [1]. At present, rotary drilling technology is the most widely used drilling method, including the rotary table drilling, the downhole power drilling and the composite drilling [2,3]. In recent years, the underbalanced drilling technology (UBD) is one of the most advanced rotary drilling techniques, including the gas phase underbalanced method, the liquid phase underbalanced method and the gas-liquid two-phase underbalanced method [4-9]. In this paper, a full factorial experiment has been performed for the conventional dry drilling of carbon fibre reinforced plastics (CFRP). The response variables are thrust force and exit-delamination by considering the drilling parameters, such as spindle speed, feed rate and point angle. Artificial neural network (ANN) has been developed to express thrust force and delamination factor as a function of drilling parameters. A new spatial modeling method is proposed for the 3D formation drill ability field. In the first stage, the number of formation modes is determined according to the formation characteristics, and these modes are identified by the fuzzy c-means clustering algorithm. In 
the second stage, random forest models are built separately for all formation modes. An infrared image segmentation algorithm combined with artificial intelligence-based technology has been carried out. The fuzzy clustering algorithm is proposed to enhance the quality of thermal images for damage assessment. A hybrid clustering-fuzzy arithmetic algorithm has been proposed by using cluster analysis to quantify porosity uncertainty. The uncertainty is projected to the irreducible water saturation and permeability by the means of fuzzy arithmetic. The proposed method is applied to five wells of the carbonate Sarvak Formation, in an Iranian onshore oil-field.

The conventional rotary drilling method cannot meet the needs of high efficiency and fast drilling. New drilling methods such as the downhole power drilling, the composite drilling and the underbalanced drilling can greatly improve the rate of penetration (ROP) and shorten the drilling cycle [10-13]. But these new drilling methods have more limitations during the application. For example, gas drilling is easily affected by the formation water and the other factors [14]. The formation with complex geological conditions often results in the interruption of drilling operation. Therefore, it is necessary to study the formation adaptability and economic applicability of different drilling methods. According to the formation characteristics, selecting a fast and economical drilling method can effectively improve the drilling speed and reduce the drilling cost.

\section{Structure}

Due to the different drilling methods and formation rock properties, the mechanism of rock breaking is also different during the drilling process. According to the mechanism of rock breaking, there are four kinds of rock breaking method, including the impact rock breaking, the cutting rock breaking, the rolling rock breaking and the grinding rock breaking [15-25].

1. Air hammer bit is a kind of rock breaking tool with the impact load. The greater the hardness and brittleness of rock, the shorter the time required for rock deformation and the greater the deformation speed. Then, the rock is easy to produce volume break, and the efficiency of rock breaking is higher. However, for the soft and plastic rock, the plastic deformation of rock has a buffer effect on the impact load. The rock breaking effect is poor and the rock breaking efficiency is low.

2. The mechanism of PDC bit is the cutting action. When the PDC bit is used to drill the soft, weak abrasive and plastic formations, the cutting teeth can continuously cut rock with the combined action of axial pressure and torque, and the rock breaking efficiency is high. But when the PDC bit is used to drill the hard, strong abrasive and brittle formations, it is difficult for PDC bit to enter the formation effectively with conventional drilling parameters, so it is necessary to increase WOB and rotate speed. This increases the friction between the bit and the rock, and it accelerates the wear of PDC bit. Therefore, the rock breaking efficiency reduces.

3. The rock breaking mechanism of cone bit is a kind of rolling action. For the soft and plastic formation, it mainly depends on the teeth to press and shear the rock under the action of dynamic and static loads. For the hard and brittle formations, the cone bit mainly relies on the impact and indentation under dynamic and static loads to break rocks. For the medium soft to medium hard strata, rock is broken mainly by the combined action of impact, indentation and shear. In general, the roller bit is widely used, and different types of bits correspond to different characteristics of the formation. The general rule of rock breaking is that the harder the formation is, the slower the $\mathrm{ROP}$ is and the lower the rock breaking efficiency is.

4. The mechanism of rock breaking is the grinding action. When the diamond with high hardness and small volume is applied to the rock, the rock produces stress concentration. It greatly exceeds the compressive strength of rock, and it results in volume breakage. At the same time, diamond with high wear resistance is often used in the hard and abrasive formations to solve the problems of low drilling efficiency and short bit life. 
Drilling fluid, drilling parameters and rock destruction are determined by the drilling methods. So the characteristics of different drilling methods should be analyzed at first.

\section{The Characteristics of Different Drilling Methods}

The drilling methods have their own characteristics and limitations. Therefore, it is necessary to understand the characteristics of different drilling methods.

1. As the most commonly used drilling method, the rotary table drilling method mainly includes the combination of the rotary table and the cone bit, the combination of the rotary table and PDC bit. However, for the difficult formations of deep and ultra-deep wells, the rotary drilling method has some problems, such as the serious energy consumption and insufficient rock breaking capacity. It cannot meet the requirements of efficient drilling.

2. The downhole power drilling method mainly relies on downhole power drilling tools to provide drilling power, and it can effectively reduce the fatigue damage of the drill string. Compared with the downhole power drilling method, the compound drilling method has the advantages of both rotary table drilling and downhole power drilling, especially in the deep wells and the difficult formations. It can greatly increase the ROP of the bit and improve the drilling efficiency.

3. During the process of the underbalanced drilling, the rock of the bottom-hole is within the negative pressure. The rock with high hardness, strong abrasiveness and high plasticity shows the properties of low hardness, weak abrasiveness and high brittleness. Therefore, the rock in the strata is easy to break. The underbalanced drilling can greatly improve the drilling speed and reduce the drilling cost. However, due to the limitation of the underbalanced drilling, especially the gas drilling method, it is necessary to predict the wellbore stability, the water yield and the downhole explosion in advance.

\section{The Fuzzy Clustering Analysis Method}

For the traditional clustering analysis, each object to be identified is strictly divided into a certain class. The boundary of the classification is clear and it belongs to the hard classification method. However, most practical problems do not have strict attribute differences, and they are intermediate in genus and morphology, and they have the same nature. In the process of long-term drilling practice, it is difficult to form a unified standard for optimizing the drilling parameters. For example, there is no necessary connection between rock abrasiveness, drill ability, drilling mode and drilling parameters. Therefore, the soft partition is more suitable. Fuzzy clustering analysis is a kind of clustering analysis method based on the fuzzy set theory. The uncertainty degree of each category can be used to represent the intermediary of sample attributes. This method can better reflect the actual problems.

\subsection{The Fuzzy Matrix}

For the fuzzy clustering analysis, the fuzzy matrix is usually used to represent the fuzzy relations over finite fields. And each fuzzy relation is corresponding to the fuzzy matrix. And fuzzy matrix is used to optimize drilling methods and drilling parameters.

When $i=1,2 \ldots m, j=1,2 \ldots n$, if $r_{i j} \in[0,1], R=\left(r_{i j}\right)_{m \times n}$ is a fuzzy matrix. The fuzzy equivalence relation of finite field $U$ can be expressed by $n \times n$ fuzzy equivalence matrix. Therefore, the operation and properties of the fuzzy matrix are similar to those of fuzzy relation.

\subsection{The Fuzzy Relation}

The fuzzy relation is the basis of the fuzzy cluster analysis. For the practical problems, the relationship between objects is often uncertain, and the fuzzy relationship can better describe the degree of fuzzy difference. There are two important operations of the fuzzy relation, including the composition of fuzzy 
relations and the equivalent operation of fuzzy relations. The establishment of the fuzzy equivalence relation is the premise of the fuzzy clustering analysis. If a fuzzy relation $R$ of the fuzzy domain $X$ satisfies the following conditions, $R$ is a fuzzy equivalence relation of the domain $X$.

1. Reflexivity: $R(x, x)=1$. Any object must be in the same class as itself.

2. Symmetry: $R(x, y)=R(y, x)$. If object $\mathrm{A}$ and object $\mathrm{B}$ are in the same class, then object $\mathrm{B}$ and object A should be in the same class.

3. Transitivity: $R \circ R \subseteq R$. If object $\mathrm{A}$ and object $\mathrm{B}$ are in the same class, and object $\mathrm{B}$ and object $\mathrm{C}$ is the same class, then object $\mathrm{A}$ and object $\mathrm{C}$ should be in the same class.

$R(x, y)$ is the degree of correlation between two objects. In general, the fuzzy relation established according to practical problems only satisfies reflexivity and symmetry, and it has no transitivity. It does not satisfy the condition of fuzzy equivalence relation. Therefore, the fuzzy similarity relation must be transformed into the fuzzy equivalence relation to realize the fuzzy clustering.

\subsection{The Transitive Closure}

For the practical problems, the fuzzy similarity relation is the most common. The transitive closure $t(R)$ of the fuzzy similarity matrix should be obtained according to the transitive closure theory. The fuzzy similar matrix is transformed into the fuzzy equivalent matrix. At present, the commonly used method to obtain transitive closure is the square method. Based on the fuzzy similar matrix $R$, the square of matrix in turn should be obtained

$R \rightarrow R^{2} \rightarrow R^{4} \rightarrow \cdots \rightarrow R^{2^{k}} \rightarrow \cdots$

When $R^{k} \circ R^{k}=R^{k}$ appears for the first time, $R^{k}$ is the transitive closure. The fuzzy similarity relation can be transformed into the fuzzy equivalence relation. Then the fuzzy classification can be realized according to the fuzzy equivalence relation.

\section{Optimization of Drilling Rock Breaking Methods Based on Fuzzy Cluster Analysis}

\subsection{The Sample Data}

Mechanical properties of rock, drilling methods, drilling parameters, logging data and many other drilling data from more than 1200 wells have been collected, such as Puguang, Yuanba and Heba blocks in Northeast Sichuan, Nanpu block in Jidong Oilfield, Luda and Jinzhou blocks in Bohai Oilfield of CNOOC. Drilling methods of adjacent wells in the same layer of different blocks are statistical analyzed. The comprehensive cost model is used to evaluate the different drilling methods. The drilling method with good effect in different layers is selected. At the same time, the logging data is collected on site, and the anti-drilling characteristics of different layers are calculated as the sample data of the fuzzy clustering analysis.

\subsection{The Clustering Index}

The drill ability, abrasiveness and plasticity coefficient of formation rock are the basic data reflecting the anti-drilling characteristics of rock. They are also an important basis for optimizing the drilling methods. The drill ability, abrasiveness and plasticity coefficient of rock are taken as the clustering index of the fuzzy clustering analysis, and the formation could be classified according to the anti-drilling characteristics. Furthermore, the purpose of the drilling mode optimization can be achieved according to the anti-drilling characteristics of formation. 


\subsection{The Data Standardization}

The sample data is the basis of the fuzzy matrix. In order to balance the role of rock drill ability, abrasiveness and plasticity in classification and eliminate the differences between them, it is necessary to standardize the sample data matrix. Then, the basic matrix of the fuzzy clustering can be obtained.

\subsection{The Similar Fuzzy Matrix}

The calculation of the similarity coefficient is the premise of establishing fuzzy similarity matrix. There are many methods to calculate the similarity coefficient between samples by using the standardized sample data. However, there is no exact principle and method to analyze the similarity of formation characteristic parameters. The angle cosine method has a unique advantage to analyze the similarity between the strata samples. It regards the stratum sample as a vector ignoring the absolute length. When the directions of two sample vectors are similar, the cosine value of the included angle is larger and the similarity is higher. On the contrary, it is lower. In this research, the angle cosine is compared with other methods during the programming process. The result showed that the distance result of using the angle cosine is more ideal. Therefore, based on the MATLAB programming calculation of fuzzy clustering (Fig. 1), the angle cosine is used to calculate the similar fuzzy matrix.

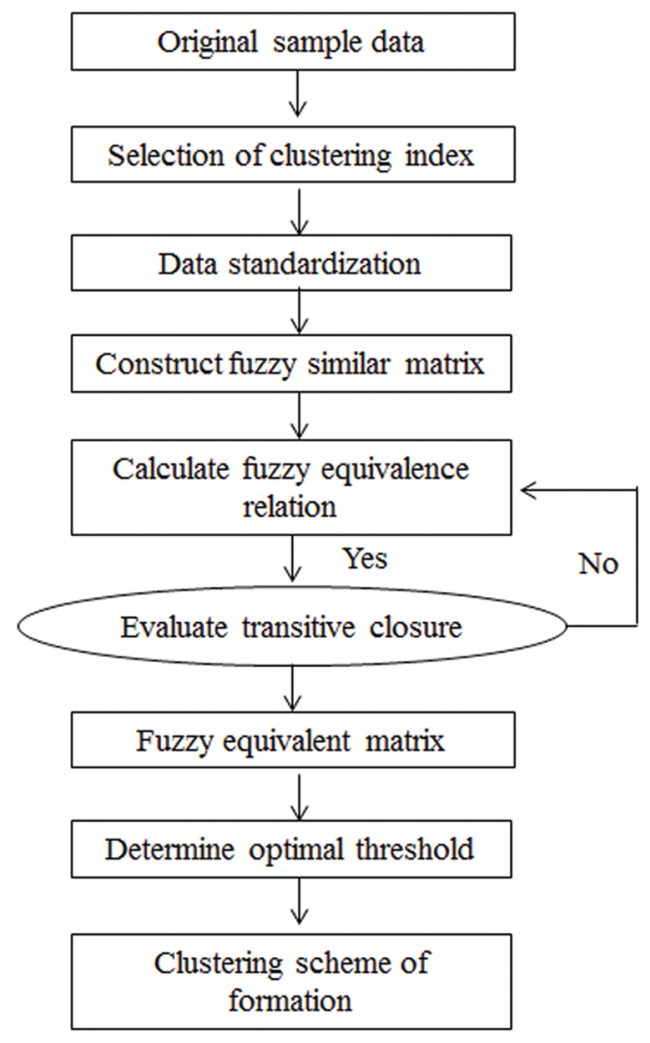

Figure 1: Fuzzy cluster analysis process

\subsection{Determination of the Fuzzy Equivalence Relation}

The fuzzy similarity matrix obtained from the sample data generally does not have the fuzzy equivalence relation. In order to classify, it must be transformed into the fuzzy equivalent matrix. By using the square method to obtain the transitive closure, the fuzzy equivalent matrix can be obtained. 


\subsection{The Optimal Classification Threshold}

The fuzzy equivalent matrix is obtained from the sample data. The different confidence level value $\lambda$ is selected to obtain the different clustering result. When $\lambda$ decreases from 1 to 0 , the clustering requirement changes from fine to course, and then it gradually merges to get the clustering pedigree. Different threshold could get different classification. According to the results of fuzzy cluster analysis and the characteristics of drilling formations, it is appropriate to divide the formations into six categories according to their anti-drilling characteristics.

$$
\begin{aligned}
& \mathrm{I}=\{1,2,3,4,5,6,7,9,16,17,18,19,20,40,41,42,43,46,47,48,50\} \\
& \mathrm{II}=\{8,10,11,12,13,21,44,45,49\} \\
& \mathrm{III}=\{14,15,22,23,24,25,30,31\} \\
& \mathrm{IV}=\{26,27,28,29\} \\
& \mathrm{V}=\{35,36,37,38,39\} \\
& \mathrm{VI}=\{32,33,34\}
\end{aligned}
$$

\subsection{The Validity Analysis of the Fuzzy Clustering}

In this research, the drilling formations are divided into six categories according to the anti-drilling characteristics. When confidence level $\lambda=0.863$ and $\alpha=0.01$, distribution table are checked to obtain $F(3,46)=4.31$. The statistic is calculated to obtain $F=14.06>4.31$. This result showed that the difference between classes is significant and the classification is reasonable.

\subsection{Analysis of Clustering Results}

Based on the analysis of the anti-drilling characteristics of different formations, the characteristics of six types of formations (Fig. 2) are summarized as follows:
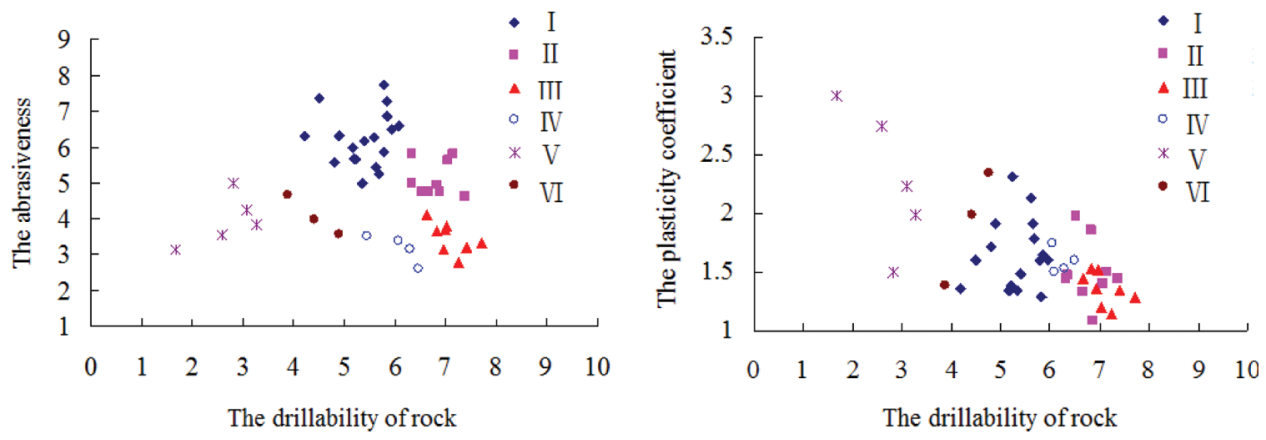

Figure 2: Statistical analysis of drilling methods of formations with different anti drilling characteristics

$\mathrm{I}=$ The drill ability is between 4.7 and 6.7. The abrasiveness is between 4.78 and 7.7. The plasticity coefficient is between 1.28 and 2.32. The drilling methods includes the underbalanced drilling method, the impregnated bit and rotary drilling, and the cone drilling.

$\mathrm{II}=$ The drill ability is between 6.67 and 7.38. The abrasiveness is between 4.6 and 6.8 . The plasticity coefficient is between 1.08 and 2.13. The drilling methods includes the underbalanced drilling method, the cone bit + rotary table drilling.

$\mathrm{III}=$ The drill ability is between 6.8 and 7.7. The abrasiveness is between 2.8 and 3.68. The plasticity coefficient is between 1.14 and 1.40. The drilling methods includes the underbalanced drilling method, the cone bit + rotary table drilling. 
IV $=$ The drill ability is between 5.73 and 6.83 . The abrasiveness is between 2.62 and 3.86 . The plasticity coefficient is between 1.50 and 1.95 . The drilling method is the combined drilling.

$\mathrm{V}=$ The drill ability is between 1.68 and 3.36. The abrasiveness is between 1.98 and 4.98 . The plasticity coefficient is between 1.5 and 3.0. The drilling method is the PDC bit + rotary table drilling.

$\mathrm{VI}=$ The drill ability is between 3.67 and 5.38. The abrasiveness is between 2.98 and 5.58. The plasticity coefficient is between 1.38 and 3.2. The drilling method is the combined drilling.

The results of the fuzzy clustering show that when the drill ability of rock is less than 5.0, the rock plasticity coefficient plays a small role in the optimization of drilling methods. The drill ability and abrasiveness of rock are the key parameters to the optimization of the drilling methods. When the drill ability of rock is more than 5.0, the drill ability, abrasiveness and plasticity of rock jointly affect the selection of drilling methods. Based on the characteristics of drilling formation division, the analysis of the characteristics of different drilling methods and the results of fuzzy cluster analysis, the criteria for optimizing drilling methods are concluded as follows:

1. For the formation with rock drill ability of $0 \sim 3.0$, the rotary table + PDC bit drilling method is recommended.

2. For the formation with rock drill ability of 3.0 5.0, when the abrasiveness is less than 6.0 , the combined drilling method is recommended.

3. For the formation with rock drill ability of 3.0 5.0, when the abrasiveness is large than 6.0 , the rotary table + cone bit drilling method or the turbo + impregnated bit method is recommended.

4. For the formation with rock drill ability of 5.0 7.0, when the abrasiveness is less than 4.0 and the plastic coefficient is large than 1.5 , the combined drilling method is recommended, especially for the deep well drilling.

5. For the formation with rock drill ability of 5.0 7.0, when the abrasiveness is less than 4.0 and the plastic coefficient is less than 1.5, the underbalanced drilling method is recommended. For the dry formation, the gas drilling method is recommended. When the formation water is less, gas drilling could be converted to the mist drilling or the foam drilling. When the formation water is serious, liquid phase underbalanced drilling is recommended. If the formation belongs to the collapsible formation, the rotary table + cone bit drilling is recommended.

6. For the formation with rock drill ability of 5.0 7.0, when the abrasiveness is large than 4.0, the underbalanced drilling method is recommended. For the dry formation, the gas drilling method is recommended. When the formation water is less, gas drilling could be converted to the mist drilling or the foam drilling. When the formation water is serious, liquid phase underbalanced drilling is recommended. If the formation belongs to the collapsible formation, the turbo + impregnated bit drilling method or the rotary table + cone bit drilling method is recommended.

7. When the drill ability of the formation is large than 7.0, the underbalanced drilling method is recommended. For the dry formation, the gas drilling method is recommended. When the formation water is less, gas drilling could be converted to the mist drilling or the foam drilling. When the formation water is serious, liquid phase underbalanced drilling is recommended. If the formation belongs to the collapsible formation, the rotary table + cone bit drilling method is recommended.

Base on the formation anti drilling characteristics, the method of optimizing the drilling method according to optimization criteria can be established. The logging data of the section to be drilled is collected firstly, then, the anti-drilling characteristics of the formation is calculated. At last, according to the established drilling method optimization criteria, the suitable drilling method suitable could be selected. The specific process is shown in Fig. 3. 


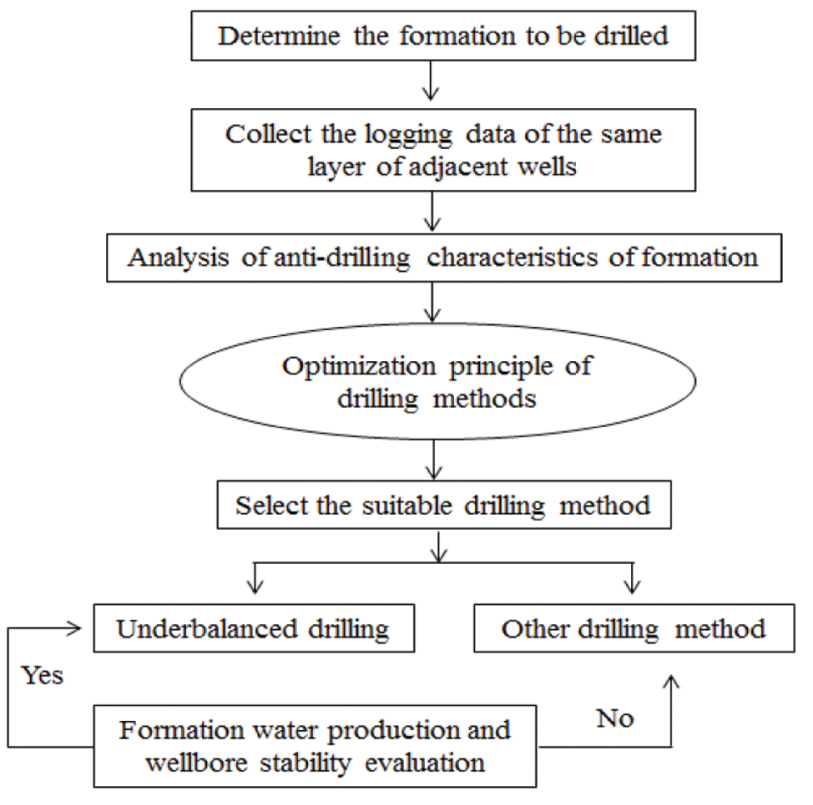

Figure 3: The flow chart of drilling method optimization

\section{Conclusions}

- Based on the classification of formation anti-drilling characteristics, the fuzzy cluster analysis method is used to analyze the statistical data. The principle of the drilling method optimization is determined.

- The optimization of drilling mode is a kind of systematic work. In this research, drilling methods are selected according to the anti-drilling characteristics of formation rocks. But there is no detailed study on the use conditions of the various drilling methods. It is suggested to carry out systematic research on the optimization of drilling methods and establish an evaluation system for the selection of drilling methods according to more characteristics of drilling formations.

Funding Statement: The authors received no specific funding for this study.

Conflicts of Interest: The authors declare that they have no conflicts of interest to report regarding the present study.

\section{References}

1. Pate, D., Thakar, V., Pandian, S., Shah, M., Sircar, A. (2019). A review on casing while drilling technology for oil and gas production with well control model and economical analysis. Petroleum, 5(1), 1-12. DOI 10.1016/j. petlm.2018.12.003.

2. Rossi, E., Jamali, S., Schwarz, D., Saar, M. O., Rohr, P. R. (2020). Field test of a combined thermo-mechanical drilling technology. Mode II: Flame-assisted rotary drilling. Journal of Petroleum Science and Engineering, 190(4), 106880. DOI 10.1016/j.petrol.2019.106880.

3. Wang, P., Ni, H., Liu, H., Zhao, B. (2020). A novel wellbore strengthening method by using rotary jet while drilling. Journal of Petroleum Science and Engineering, 194(6), 107556. DOI 10.1016/j.petrol.2020.107556.

4. Fan, X., Zhang, Q., Wang, J., Xu, L. (2015). Mathematical methods for evaluating a reservoir based on gas dynamic monitoring during underbalanced drilling. Journal of Natural Gas Science and Engineering, 26(10), 1068-1079. DOI 10.1016/j.jngse.2015.07.035. 
5. Abdelghany, W. K., Radwan, A. E., Elkhawaga, M. A., Wood, D. A., Sen, S. et al. (2021). Geomechanical modeling using the depth-of-damage approach to achieve successful underbalanced drilling in the Gulf of Suez rift basin. Journal of Petroleum Science and Engineering, 202(8), 108311. DOI 10.1016/j.petrol.2020.108311.

6. Wang, Q., Jia, X. (2020). Multi-objective optimization of CFRP drilling parameters with a hybrid method integrating the ANN, NSGA-II and fuzzy C-means. Composite Structures, 235(1-4), 111803. DOI 10.1016/j. compstruct.2019.111803.

7. Gan, C., Cao, W., Liu, K., Wu, M. (2021). A new spatial modeling method for 3D formation drillability field using fuzzy c-means clustering and random forest. Journal of Petroleum Science and Engineering, 200(10), 108371. DOI 10.1016/j.petrol.2021.108371.

8. Wang, Z., Wan, L., Xiong, N., Zhu, J., Ciampa, F. (2021). Variational level set and fuzzy clustering for enhanced thermal image segmentation and damage assessment. NDT \& E International, 118(8), 102396. DOI 10.1016/j. ndteint.2020.102396.

9. Masoudi, P., Aïfa, T., Memarian, H., Tokhmechi, B. (2018). Uncertainty assessment of porosity and permeability by clustering algorithm and fuzzy arithmetic. Journal of Petroleum Science and Engineering, 161(2), 275-290. DOI 10.1016/j.petrol.2017.11.018.

10. Nwanwe, O. I., Teodoriu, C. (2020). Matrix selection and comparison for selecting drilling methods and technologies for a wide range of applications. Journal of Petroleum Science and Engineering, 192(5), 107289. DOI 10.1016/j.petrol.2020.107289.

11. Wang, X., Ni, H., Shor, R., Wang, R. (2021). A model-based optimization and control method of slide drilling operations. Journal of Petroleum Science and Engineering, 198(6), 108203. DOI 10.1016/j.petrol.2020.108203.

12. Jiang, H., Liu, G., Li, J., Zhang, T., Wang, C. (2021). A realtime drilling risks monitoring method integrating wellbore hydraulics model and streaming-data-driven model parameter inversion algorithm. Journal of Natural Gas Science and Engineering, 85(7), 103702. DOI 10.1016/j.jngse.2020.103702.

13. Wang, C., Liu, G., Yang, Z., Li, J., Zhang, T. et al. (2020). Downhole working conditions analysis and drilling complications detection method based on deep learning. Journal of Natural Gas Science and Engineering, 81(8), 103485. DOI 10.1016/j.jngse.2020.103485.

14. Lan, P., Polycarpou, A. A. (2020). Stribeck performance of drilling fluids for oil and gas drilling at elevated temperatures. Tribology International, 151(2), 106502. DOI 10.1016/j.triboint.2020.106502.

15. Wu, Z., Yu, F., Zhang, P., Liu, X. (2019). Micro-mechanism study on rock breaking behavior under water jet impact using coupled SPH-FEM/DEM method with Voronoi grains. Engineering Analysis with Boundary Elements, 108(1), 472-483. DOI 10.1016/j.enganabound.2019.08.026.

16. Zhu, X., Luo, Y., Liu, W. (2020). On the rock-breaking mechanism of plasma channel drilling technology. Journal of Petroleum Science and Engineering, 194(2), 107356. DOI 10.1016/j.petrol.2020.107356.

17. Chukhrova, N., Johannssen, A. (2019). Fuzzy regression analysis: Systematic review and bibliography. Applied Soft Computing, 84(3), 105708. DOI 10.1016/j.asoc.2019.105708.

18. Qiu, Q., Kou, M., Chen, K., Deng, Q., Lin, C. (2020). Optimal stopping problems for mission oriented systems considering time redundancy. Reliability Engineering \& System Safety, 205, 107226. DOI 10.1016/j. ress.2020.107226.

19. Okubo, S., Fukui, K., Nishimatsu, Y. (2018). Estimating abrasivity of rock by laboratory and in situ tests. Rock Mechanics and Rock Engineering, 44(2), 231-244. DOI 10.1007/s00603-010-0113-6.

20. Mohamad, E. T., Saad, R., Hamzah, N. N. B., Akip Tan, S. N. M., Liang, M. (2012). Assessment on abrasiveness of rock material on the wear and tear of drilling tool. Electronic Journal of Geotechnical Engineering, 17, 91-100.

21. Šolc, T., Stefanovska, A., Hoey, T., Mikoš, M. (2019). Application of an instrumented tracer in an abrasion mill for rock abrasion studies. Journal of Mechanical Engineering, 58(4), 263-270. DOI 10.5545/sv-jme.2010.215.

22. Kaesling, H., Thuro, K. (2010). Determining rock abrasivity in the laboratory. Rock Mechanics in Civil and Environmental Engineering-Proceedings of the European Rock Mechanics Symposium, pp. 425-428.

23. Qiu, Q., Cui, L. (2019). Gamma process based optimal mission abort policy. Reliability Engineering \& System Safety, 190(2), 106496.1-106496.9. DOI 10.1016/j.ress.2019.106496. 
24. Oparin, V. N., Tanaino, A. S. (2019). Assessment of abrasivity by physico-mechanical properties of rocks. Journal of Mining Science, 45(3), 240-249. DOI 10.1007/s10913-009-0031-8.

25. Ba, Q., Liu, Y., Zhang, Z., Xiong, W., Shen, K. (2021). Analysis of the flow field characteristics associated with the dynamic rock breaking process induced by a multi-hole combined external rotary bit. Fluid Dynamics \& Materials Processing, 17(4), 697-710. DOI 10.32604/fdmp.2021.014762. 